\title{
Self-management interventions for skin care in people with a spinal cord injury: part 1-a systematic review of intervention content and effectiveness
}

\author{
Justine S. Baron ${ }^{1} \cdot$ Katrina J. Sullivan ${ }^{1} \cdot J_{i l l i a n}$ M. Swaine $e^{2,3} \cdot$ Arlene Aspinall $^{4,5} \cdot$ Susan Jaglal ${ }^{6,7} \cdot$ Justin Presseau $^{1,8}$. \\ Barry White $^{4} \cdot$ Dalton Wolfe $^{9,10} \cdot$ Jeremy M. Grimshaw ${ }^{1,11}$
}

Received: 19 November 2017 / Revised: 2 April 2018 / Accepted: 4 April 2018 / Published online: 25 May 2018

(c) The Author(s) 2018. This article is published with open access

\begin{abstract}
Study design Systematic review.

Objectives To review the content and effectiveness of skin care self-management interventions for people with SCI.

Setting International.

Methods We searched electronic bibliographic databases, trial registers, and relevant reference lists. Eligibility criteria for the reviews of intervention content and effectiveness were identical with the exception of study design. The review of intervention content included non-randomized and randomized controlled trials (RCTs). The review of effectiveness included RCTs. A Behavior Change Technique (BCT) taxonomy of 93 BCTs was used to code intervention content. Intervention effects on outcomes of interest are summarized descriptively. Effect sizes were calculated, and the Cochrane risk of bias tool applied. Results In all, 15 studies testing 17 interventions were included in the review of intervention content. Interventions in these studies included 28 BCTs. The most common were "instructions on how to perform behavior" (16 interventions), "credible source" (12 interventions), and "social support (unspecified)" (9 interventions). Ten RCTs were included in the review of intervention effectiveness and they measured knowledge, self-efficacy, and skills relating to skin care/pressure ulcer (PU) prevention, skin care behaviors, skin status (PU prevalence, severity, and time to PU), and health-care utilization for skin problems. Evidence to support intervention effects on these outcomes was limited, particularly for clinical outcomes. Risk of bias assessments was often inconclusive due to poor reporting.

Conclusions There is potential to design SCI skin care interventions that include currently untested BCTs. Further research and better consistency in outcome measurements and reporting are required to synthesize evidence on effectiveness.
\end{abstract}

Electronic supplementary material The online version of this article (https://doi.org/10.1038/s41393-018-0138-3) contains supplementary material, which is available to authorized users.

$\triangle$ Justine S. Baron

justinebaron@gmail.com

1 Clinical Epidemiology Program, Ottawa Hospital Research Institute, Ottawa, ON, Canada

2 Faculty of Health and Medical Sciences, University of Western Australia, Perth, WA, Australia

3 Institute for Health Research, University of Notre Dame Australia, Fremantle, WA, Australia

4 Rick Hansen Institute, Vancouver, BC, Canada

5 Vancouver General Hospital, Vancouver, BC, Canada
6 Department of Physical Therapy, University of Toronto, Toronto, ON, Canada

7 Toronto Rehabilitation Institute, Toronto, ON, Canada

8 School of Epidemiology and Public Health, University of Ottawa, Ottawa, ON, Canada

9 Parkwood Institute Research, Lawson Health Research Institute, London, ON, Canada

10 University of Western Ontario, London, ON, Canada

11 Department of Medicine, University of Ottawa, Ottawa, ON, Canada 


\section{Introduction}

Pressure ulcers (PUs; or pressure injuries) are among the commonest secondary complications affecting people with a spinal cord injury (SCI) living in the community. Period prevalence over 3 months has been found to be as high as $34.7 \%$ [1]. PUs lead to high rates of health-care utilization following discharge from SCI rehabilitation, and one PU adds an average of $\$ 18,758$ to hospital admission costs in Canada [2]. They result in significant social (e.g., isolation) and financial (e.g., unemployment) limitations, as well as psychological difficulties (e.g., negative emotions) [3].

One way to help prevent PUs in the SCI community is by influencing modifiable risk factors, such as patients' selfcare behaviors. Preventive skin care is less than optimal in the SCI community, with one study suggesting that $29.9 \%$ of PUs are associated with self-care behaviors [4]. Some skin care behaviors are commonly performed by people with SCI (e.g., skin care in case of incontinence and examination of the cause of PUs). Others such as daily skin checks and pressure relief are performed by $50 \%$ or fewer [5]. Similarly, less than 5\% of people with SCI adhere to dietary recommendations [6], 37\% are inactive [6], and 30\% delay visiting a physician after detecting a PU [7].

Self-management interventions for people with SCI have been designed to reduce non-adherence to preventive skin care. Three reviews [8-10] have synthesized this work, but they focus only on educational interventions, technologybased interventions, or behavioral and educational interventions with a primary focus on PU prevention. None of these reviews has used a systematic approach to describe intervention content. Systematically describing intervention content is an important step in building a cumulative science [11]. The aims of this study were to: (1) review the active ingredients in self-management interventions for skin care in SCI and (2) review the effectiveness of these same interventions. A separate publication [12] reports on theory use and reporting quality.

\section{Methods}

We published a protocol [13] prior to undertaking this review. Common search and study selection procedures were used to address aims 1 and 2. Eligibility criteria for these aims were also identical, with the exception of study design. A greater number of study designs were eligible to address aim 1 as this work was performed to better understand the content of the interventions designed in this area of research. In contrast, the focus on effectiveness in aim 2 resulted in restricting study designs to randomized controlled trials (RCTs).

\section{Search strategy}

\section{Bibliographic databases}

A MEDLINE search strategy was designed to include search terms on SCI, self-management, and skin care. The MEDLINE search strategy was peer-reviewed by an independent librarian using the Peer-Review of Electronic Search Strategy checklist [14]. In consultation with the librarian, it was adapted for use in four other large electronic databases (Embase, PsycINFO, CENTRAL, and CINAHL). Smaller databases (REHABDATA, CIRRIE, PeDro, and ERIC) were searched using keywords and subject headings if available. All search strategies were run on 23 February 2016 and are presented in Supplementary Information 1.

\section{Additional data sources}

Relevant posters, abstracts, and conference proceedings identified via the electronic bibliographic database search were used to search for papers. In addition, reference lists of relevant published protocols, systematic reviews, and of the final list of included studies were hand-searched. Authors were contacted for further information and publications. Authors announcing a forthcoming publication were recontacted in March 2017 to identify accepted or published papers.

Trial registers (World Health Organization International Clinical Trials Registry and Meta-Register of Controlled Trials) were searched on 21 June 2016 using keywords (see Supplementary Information 1). Publications related to relevant studies were searched. If none were found, principal investigators were contacted for information and publications.

\section{Eligibility criteria}

To address aim 1 (review of the content of skin care selfmanagement interventions for people with SCI), RCTs and non-randomized trials with a control group receiving standard care and published in English were included. Studies must have primarily involved people with SCI (representing $50 \%$ or more of the sample). Included studies tested interventions that addressed, at least in part, skin care self-management capabilities related to PU prevention. Given that multiple behaviors are often addressed to varying degrees in SCI self-management interventions, studies needed to include measurement of at least one of the following outcomes of interest: mediators of skin care behaviors (e.g., self-efficacy or skills, and knowledge relating to skin care or PU prevention), skin care behaviors, and PU-related clinical outcomes (e.g., PU prevalence, incidence, re-occurrence, or severity). Studies 
with a primary focus on PU treatment were excluded, as were studies targeting more lifestyle-related behaviors (e.g., improving nutritional intake or physical activity and smoking cessation) that can affect physiological indicators of skin health. No exclusion criteria were applied with respect to intervention delivery setting (e.g., inpatient, outpatient, and community), length of follow-up, or publication date.

The same eligibility criteria were applied to address aim 2 (review on effectiveness), with the exception of study design that was restricted to RCTs.

\section{Study selection}

Two reviewers (J.S.B. and J.M.S.) independently screened titles and abstracts of bibliographic database search results. Articles not meeting inclusion criteria were removed. Full texts of remaining publications were then independently reviewed.

J.S.B. was responsible for the identification of potentially relevant papers using additional data sources. J.M.S. screened papers identified as potentially relevant based on their titles and abstracts. Both reviewers then independently screened full texts.

Discrepancies in screening outcomes were discussed until consensus was reached. A third reviewer (J.M.G.) was consulted in case of disagreement.

\section{Data extraction}

A data extraction spreadsheet was designed in Excel to capture general information, study characteristics, participants, intervention characteristics, measurements, data analyses, and intervention effects (see protocol [13]). It was piloted on two papers reporting SCI behavioral interventions and ineligible for inclusion in this review.

Two reviewers (J.S.B. and K.J.S.) independently extracted data. Discrepancies were discussed until consensus was reached, or a third party was consulted (J.M.G.).

\section{Aim 1: Review of intervention content (RCTs and non-RCTs)}

Data extraction for intervention content was based on intervention and control group treatment descriptions in eligible papers, and in any related papers and intervention materials located by reviewers (e.g., cited in text) or during correspondence with authors. These materials could include published protocols, intervention pilots, intervention development papers, and any unpublished document supporting intervention delivery (e.g., intervention manuals, scripts, and PowerPoint slides). Intervention components delivered to SCI participants were coded into behavior change techniques (BCTs) [11], defined as the smallest observable and replicable components of behavioral interventions designed to bring about change. The BCT taxonomy [11] includes 93 BCTs (see Supplementary Information 2). It comes with online training, which both coders completed. Our protocol suggested that we would also use a second, broader taxonomy of self-management components [15]. Results using this broader taxonomy were not considered to add value to the more precise BCT taxonomy codes and have, therefore, not been reported here (available upon request).

\section{Aim 2: Review of intervention effectiveness (RCTs only)}

We examined effectiveness of interventions tested in RCTs, and focused on extracting data for time points where intervention and control group participants were assessed. It was anticipated that a meta-analysis would not be feasible, given our preliminary knowledge of the varied outcome measures in SCI self-management studies. Intervention effects are described, classified by outcome type (mediators of skin care, skin care behaviors, and clinical outcomes) but are not statistically pooled.

We aimed to present effect sizes and 95\% confidence intervals (CIs) where sufficient data were available and for studies with samples larger than 10 participants. Hedges' $g$ [16] effect size was used for continuous outcomes and odds ratio (OR) was used for categorical data. Hedges' $g$ is interpreted the same way as Cohen's $d(0.2=$ small, $0.5=$ medium, and $0.8=$ large). Data pertaining to the longest follow-up were used for these effect size calculations. We report continuous outcomes as a percentage change from baseline and as a mean score at follow-up if both were provided, but the effect size was computed using mean scores. When scale and individual item scores were available for the same outcome, the former were used for effect size calculations. Authors were contacted when outcome data were incomplete or unclear.

To complement our assessment of effectiveness with one of study quality, two reviewers (J.S.B. and K.J.S.) independently assessed risk of bias using the Cochrane tool [17]. This tool focuses on random sequence generation, allocation concealment, blinding of study personnel and outcome assessors, incomplete outcome data, selective reporting, and other sources of bias (rated as "low risk", "high risk", or "unclear risk" of bias). The domain blinding of outcome assessors was split into two items, one for mediators of behavior and the second for PU-related clinical outcomes, and studies that did not include these outcomes were rated as "Not Applicable". Disagreements between reviewers were resolved by discussion. A third author (J.G.) was consulted for arbitration. 
Fig. 1 PRISMA flow diagram. Systematic review of skin care self-management interventions for people with SCI

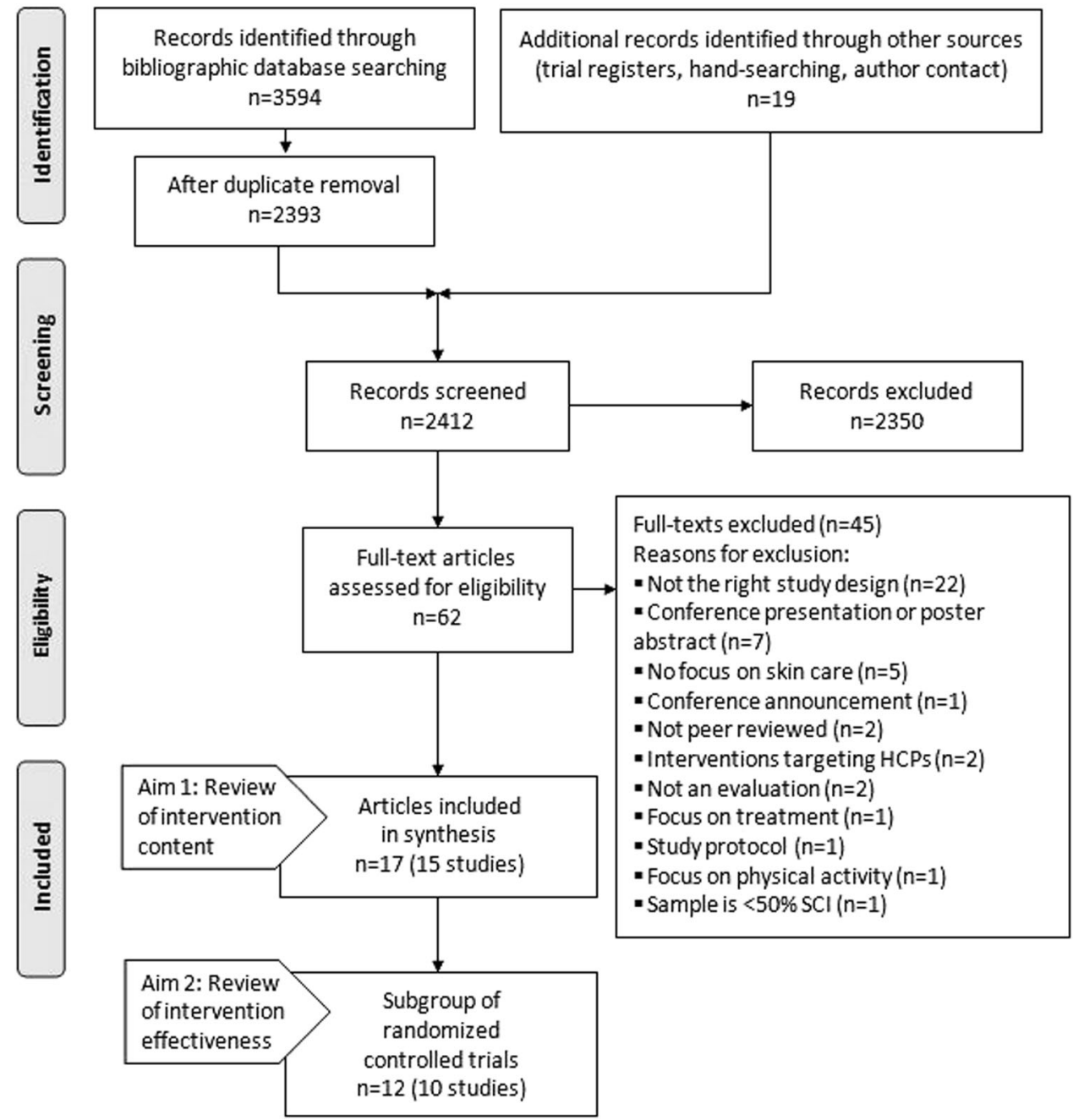

\section{Results}

Figure 1 is a PRISMA flow diagram. Electronic bibliographic databases and additional data source searching returned 2412 papers. Following title and abstract screening, 62 were screened in full text. Seventeen were eligible for the review on intervention content (aim 1). A list of excluded studies is available in Supplementary Information 3. These 17 papers described 15 studies, 10 of which were RCTs and therefore included in the review on intervention effectiveness (aim 2). Publications used to maximize data extraction included a published protocol [18], intervention development or pilot test publications [19-21], and one erratum [22].

\section{Aim 1: Review of intervention content (RCTs and non-RCTs)}

The 15 studies reviewed tested 17 different interventions. Fifteen control groups received standard care consisting in very limited or no contact with health-care professionals
(HCPs) or intervention deliverers (10 studies [23-33]), and minimal education (five studies [22, 34-38]). A description of intervention and control group treatments is in Table 1 .

The interventions tested in the 15 studies can be classified into five categories according to their primary component and mode of delivery, and irrespective of BCTs:

1. Structured education programs (five studies $[22,32$, 34-37] including two RCTs [22, 34, 36]): Interventions consisted of educational sessions that were predefined in content and delivered to individuals (one study [22, 36]) or groups (four studies [32, 34, 35, 37]). Follow-up phone calls to deliver behavior reminders and motivational interviewing were included in two studies [22, 34, 36].

2. Telehealth (four studies [24, 25, 28, 29, 33] including three RCTs [24, 25, 29, 33]): These interventions were primarily delivered to individuals using videoor phone-based technologies. Participants were provided with health education, monitoring of skin health 


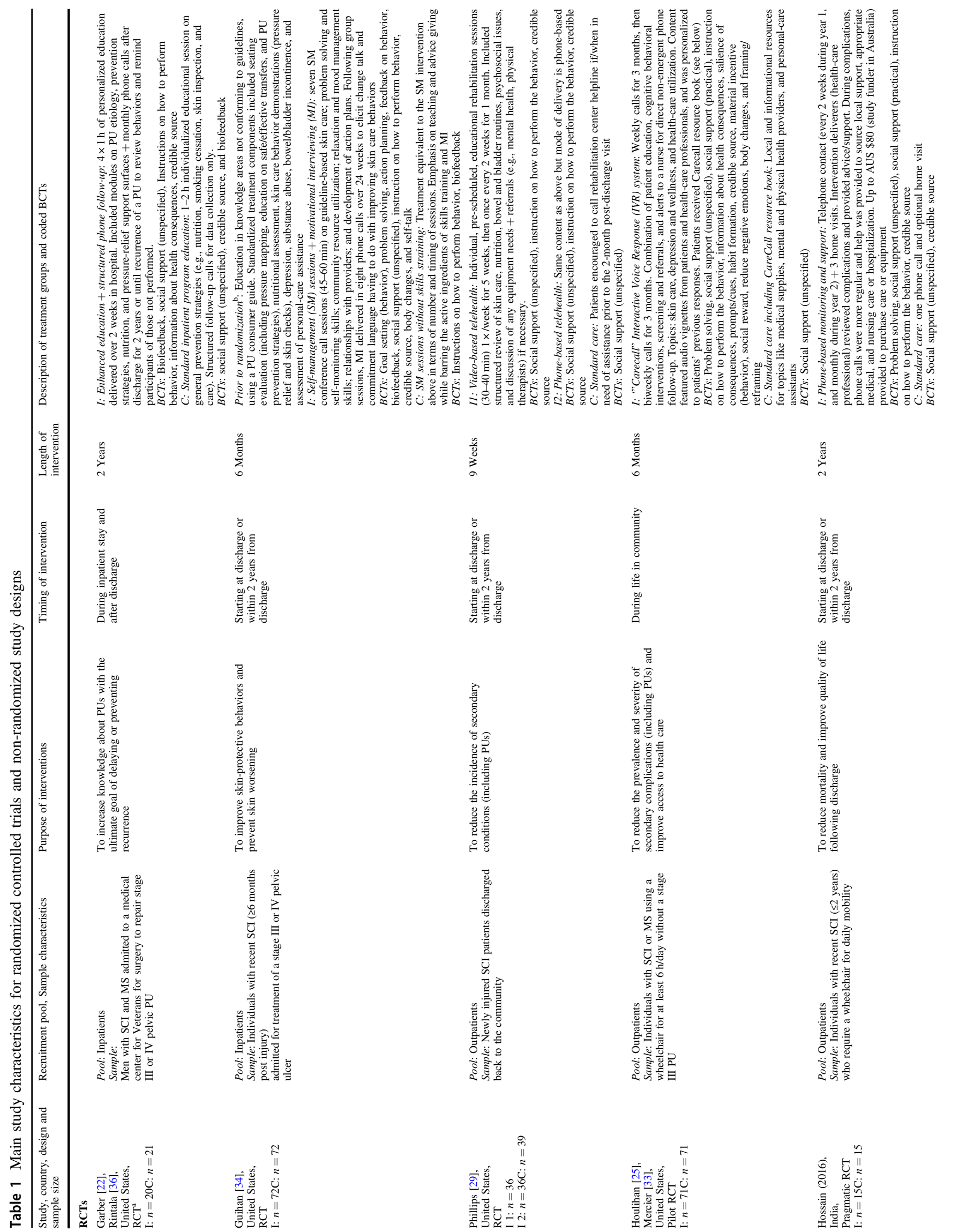




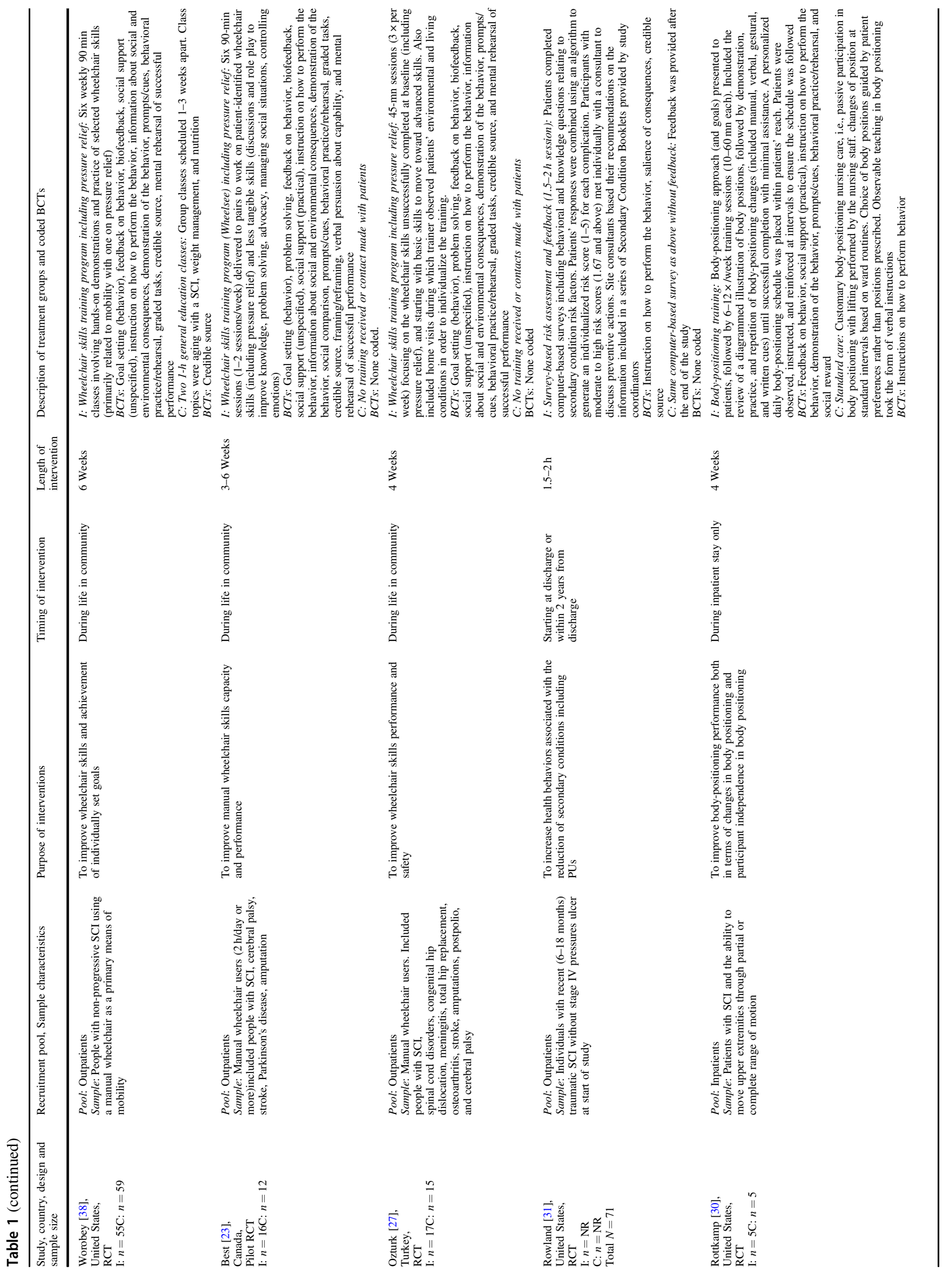




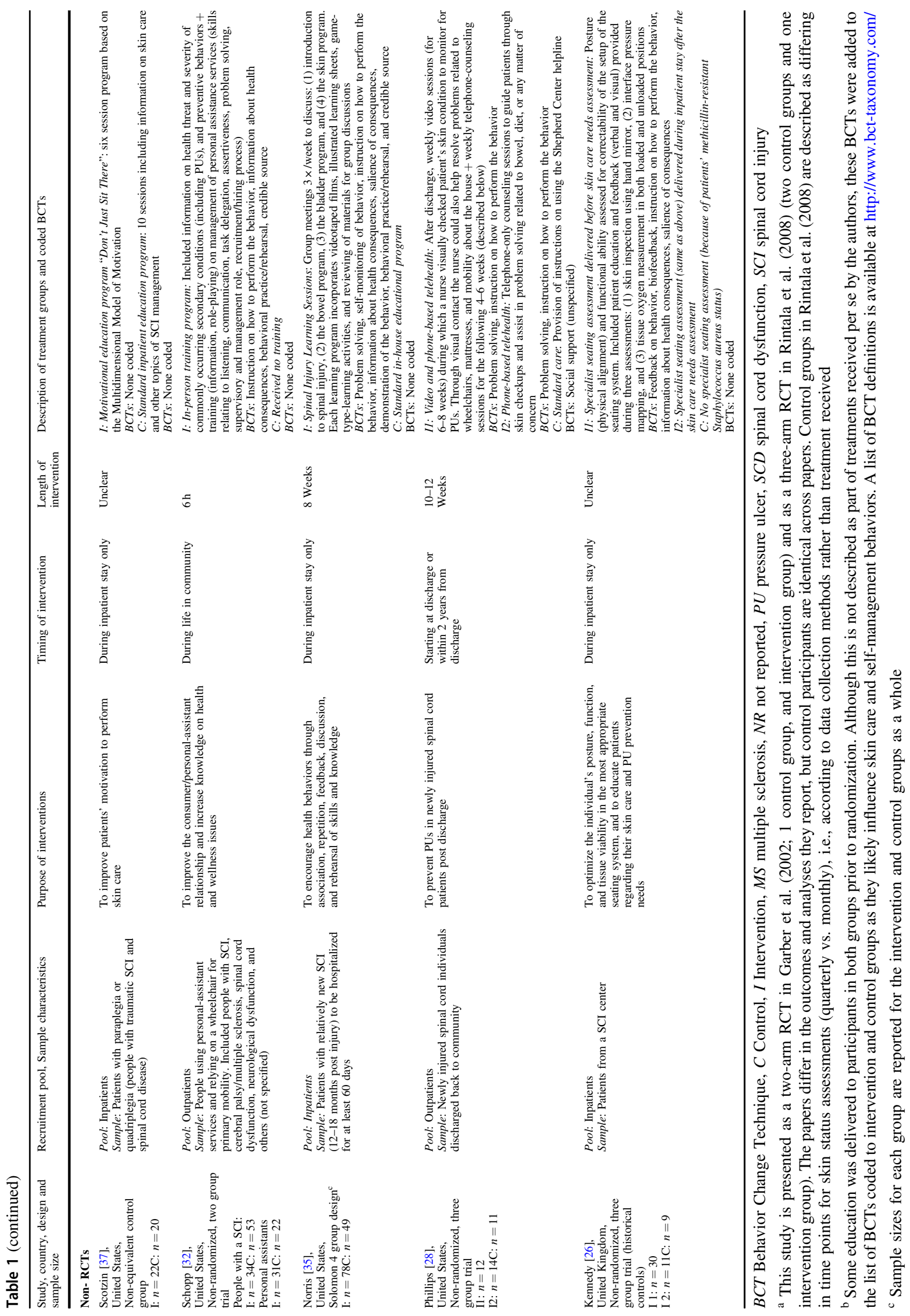


by study personnel, guidance on skin care behaviors, a review of their needs by a $\mathrm{HCP}$, and/or advice and support to manage SCI complications including PUs (if necessary).

3. Wheelchair skills training (three RCTs [23, 27, 38]): These studies evaluated the same Wheelchair Skills Training program that aimed to improve participants' performance of a range of wheelchair skills. The majority of wheelchair skills were related to mobility and ability to navigate their environment. A smaller proportion of skills related to pressure relief for skin care ("relieving weight from buttocks").

4. Risk assessment and feedback (two studies [26, 31] including one RCT [31]): These interventions made the risks associated with certain body positions and behaviors salient. One study [26] provided participants with visual feedback on the effects of pressure distribution. The second study [31] consisted of providing automated (i.e., computer-generated) and in-person feedback on the risks associated with some behaviors in the prevention of secondary complications including PUs.

5. Body-positioning skills training (one RCT [30]): This intervention encouraged participants to change body position through demonstration, practice, reinforcement, and personalized guidance on pressure relief frequency.

The BCT taxonomy was applied to all 17 interventions (k) and 15 controls (c) group treatment descriptions. Intervention materials were used to support coding of seven $[22,23,25,27,33-36,38](42 \%)$ of the 17 interventions reviewed (see Supplementary Information 4 for a list of used and missing intervention materials). Reasons for nonavailability of intervention materials included authors no longer having copies of materials, lack of corresponding email addresses to contact authors, author preference to keep documents confidential, and author non-response.

One study [34] included the delivery of care components before randomization (i.e., instructions on how to perform skin care and biofeedback). As these were likely to influence skin care and PU outcomes, a decision was made to code them for both intervention and control group treatments. In four other studies [23, 27, 35, 38], the intervention materials used to code BCTs provided a full description of a program. It remained unclear whether the full program was implemented, but none of the authors reported adjustments or tailoring to local context.

On average, $12.3 \pm 4.0 \mathrm{BCTs}$ (range $8-17$, median 12) were coded in the seven interventions for which intervention materials were available. In these, $6.4 \pm 2.1$ BCT codes (range 2-8, median 7) were unique to intervention materials (i.e., absent from intervention descriptions in published papers). For the 10 interventions for which no supporting materials were available, the average number of BCTs was $3.3 \pm 1.8$ BCTs (range 0-6, median 3). For control group treatments, the average number of BCTs coded was $0.8 \pm$ 0.9 (range 0-3, median 1).

The 17 interventions included 28 (30\%) of the 93 BCTs listed in the BCT taxonomy (see Fig. 2 for a graphical display, and Supplementary Information 5 for the studies they apply to). The three most commonly coded BCTs were instructions on how to perform behavior $(k=16,94 \%)$, credible source (interventions where verbal or visual communications in favor of or against the behavior are presented by a credible source, e.g., HCP; $k=12,71 \%$ ), and social support (unspecified; $k=9,53 \%$ ).

Four BCTs were coded across the 15 control group treatment descriptions (see Supplementary Information 6): social support (unspecified; $c=5,33 \%)$, credible source ( $c$ $=3,20 \%)$, instructions on how to perform behavior $(c=2$, $13 \%)$, and biofeedback ( $c=2,13 \%)$. The small number of BCTs coded reflects the low level of intervention associated with standard care, as well as the lack of description of control group treatment [39] (see Table 1).

\section{Aim 2: Review of intervention effectiveness (RCTs only)}

Table 1 includes detailed information on key characteristics of the 10 RCTs [22-25, 27, 29-31, 33, 34, 36, 38]. Just over half of RCTs (six studies [24, 29-31, 34, 38]) were specific to people with SCI, and half (five studies [22-24, 27, 30, 36]) included a sample size of 50 participants or less. The shortest intervention length was $2 \mathrm{~h}$ in one RCT [31], and the longest was 2 years in two RCTs [22, 24, 36]. Only three interventions were delivered during life in the community (no association with an inpatient stay) [23, 27, 38]. Intervention groups in RCTs included 26 BCTs, the most common being identical to those listed above.

\section{Risk of bias}

Figure 3 is a graphical display of overall risk of bias in the 10 RCTs [22-25, 27, 29-31, 33, 34, 36, 38]. Risk of bias results for each study can be found in Supplementary Information 7. Only three studies were judged not to have a high risk of bias in any of the seven domains evaluated [29-31]. Blinding of participants and personnel, and incomplete outcome data reporting were the two risk of bias domains most frequently rated "high risk". A large proportion of the risk assessment domains could not be rated because of incomplete or unclear information ("unclear"). Allocation concealment was the domain with the greatest number of "unclear" ratings. 


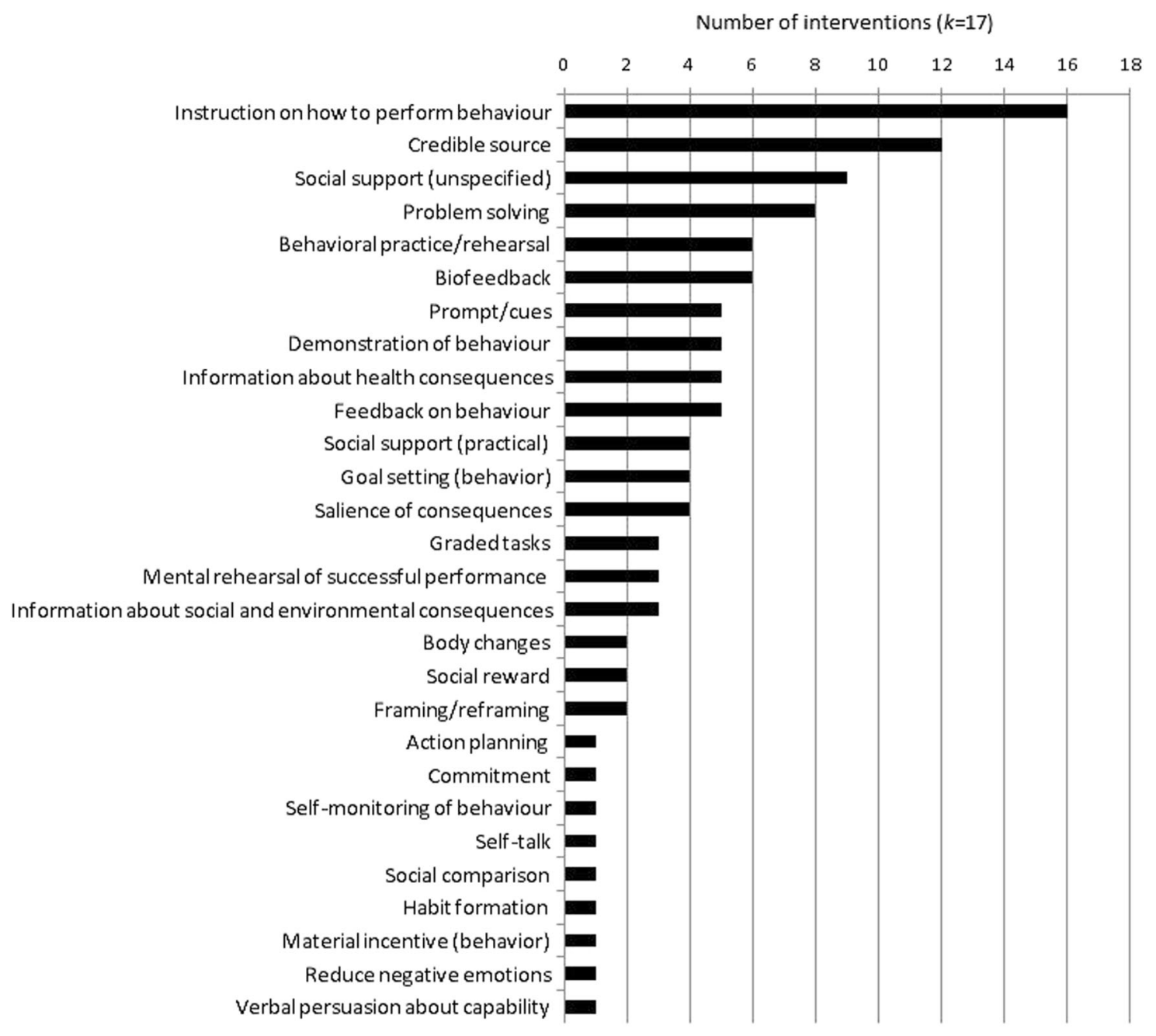

Fig. 2 Behavior change techniques coded to the 17 interventions reviewed

\section{Intervention effects on outcomes}

A narrative summary of findings is presented below by outcome measure, with Supplementary Information 8 detailing study findings. Effect sizes could be calculated in four studies. They are included below.

\section{Mediators of skin care behaviors}

Mediators of skin care measured included knowledge, selfefficacy, or skills relating to skin care.

\section{Knowledge about skin care and PU prevention}

A self-report measure of knowledge was included in three studies [22, 31, 34, 36]. Two [22, 34, 36] studies used the same Pressure Ulcer Knowledge test (unclear psychometric properties). Complete reporting of knowledge data was available in one study $[22,36]$ that found that an enhanced educational inpatient program including $4 \mathrm{~h}$ of individualized learning sessions resulted in significantly greater knowledge about PUs compared to a short education program delivered over 1-2 $\mathrm{h}$. The effect size (Hedges' $g$ ) was 0.71 (95\% CI 0.06, 1.36).

\section{Self-efficacy relating to skin care}

Self-efficacy for skin care (i.e., confidence in one's ability to prevent and manage PUs) was measured in one study [34] using a validated scale adapted for PUs. No data were reported or provided by authors.

\section{Skills relating to skin care}

Four studies assessed skills relating to skin care [23, 27, 30, 38]. Three studies [23, 27, 38] testing a wheelchair skills training program included a trainer administering a validated Wheelchair Skills Test Questionnaire, which involves the assessment of 


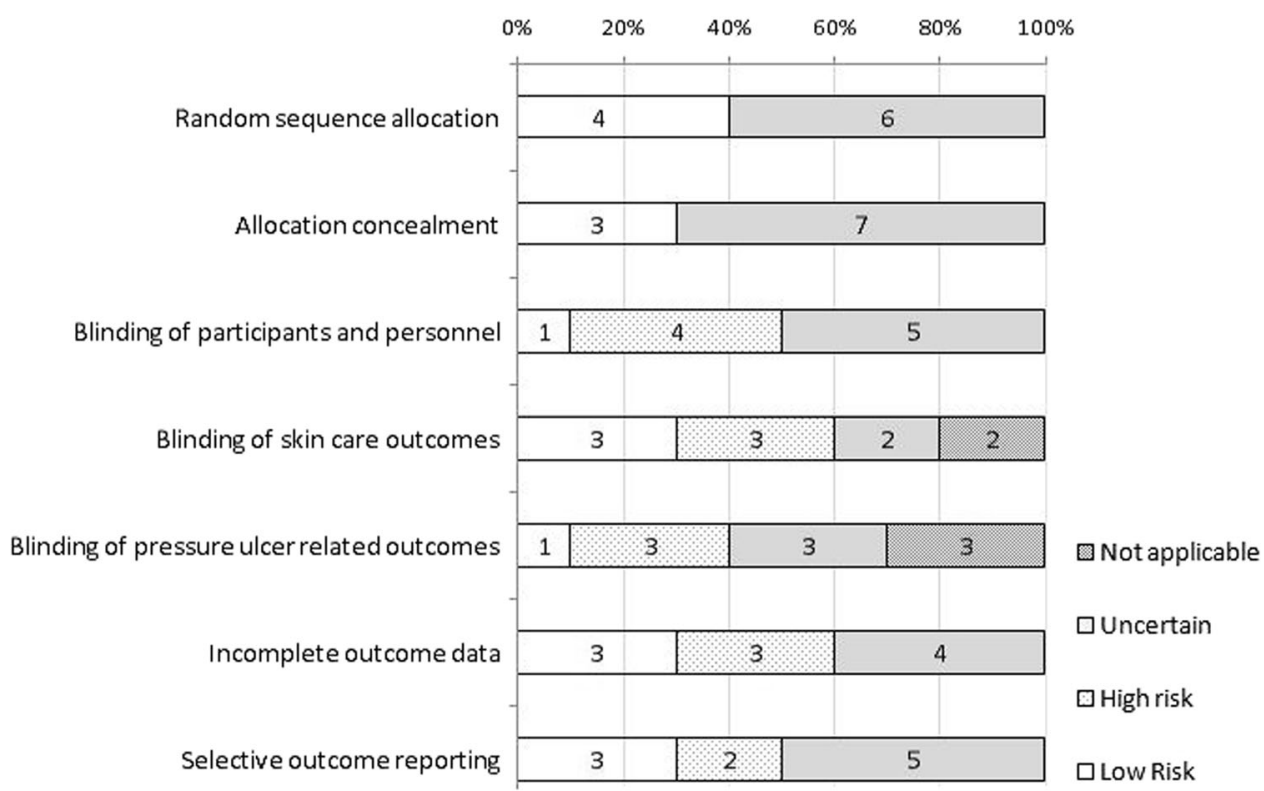

Fig. 3 Risk of bias assessment results across the 10 randomized controlled trials reviewed. Notes: High high risk of bias, low low risk of bias, Uncertain unclear risk of bias. The "unclear" category was used where there was insufficient detail for a conclusion to be reached, or where there was no published/registered study protocol available (item on selective outcome reporting). A decision about the selective

over 30 individual wheelchair skills including one item on pressure relief (ability to "relieve pressure from buttocks"). Individual scores for the pressure relief skill were not reported in any of these studies. Although significant improvements in overall wheelchair skill summary scores were observed for the intervention group in all three studies [23, 27, 38], none of the participants worked on the pressure relief skill. This was by choice in two studies [23, 38], or the result of successfully completing this skill at baseline in the third study [27] (Ozturk A., personal communication, 14 February 2017).

A fourth study [30] that measured skills tested a 4-week inpatient body-positioning training program in which patients were taught how to change body position, and received social reinforcement and encouragements to follow an individualized body-positioning schedule. An HCP assessed the degree of assistance required for body change positions, using a self-developed scale with face validity. A larger decrease in assistance needed was observed in intervention participants at 4 weeks compared to controls. The sample size, however, was small $(n=10)$.

\section{Skin care behaviors}

Four studies included a behavioral measure of skin care [25, 30, 31, 33, 34]; however, data were only available for three studies [25, 30, 33, 34]. Behavioral measures included items relating to skin checks, pressure relief (e.g., frequency outcome reporting item was reached by comparing outcomes reported on to measures outlined in registered or published protocols. If a protocol was not available, this item was marked as "unclear". In addition, items on blinding of outcomes were rated "not applicable" if skin care outcomes or pressure-ulcer-related outcomes were not measured

of body position changes and intervals of prolonged skin pressure), wheelchair cushion checks, alcohol and substance abuse, help seeking with new or worsening skin breakdown, and keeping skin clean and dry. The two studies [30, 34] for which between-group differences were tested statistically yielded mixed findings. The first study [34] compared a self-management and skills training (problem solving, selfmonitoring, and mood and stress management) intervention plus motivational interviewing follow-up phone calls to a standard care self-management intervention without skill training or motivational interviewing. The proportion of skin care behaviors performed (measured using a selfdeveloped Skin Care Behavior Checklist with unclear psychometric properties) did not significantly increase at 3 and 6 month follow-ups in the intervention group compared to controls, and the effect size CIs crossed zero (Hedges' $g=$ $0.13,95 \% \mathrm{CI}-0.19,0.46)$. In contrast, results of the second study [30] $(n=10)$ indicated that a 4-week inpatient bodypositioning training program increased the frequency of pressure relief behaviors measured via HCP observations. Finally, descriptive statistics performed on the raw data sent to our research team for a third study [25, 33] evaluating a telehealth intervention (Houlihan B., personal communication, 3 April 2017) suggested that the greate st group differences at follow-up were on two pressure relief items (sitting and in bed), with the intervention group performing pressure relief more frequently. The effect size calculated 
using the total scale score indicated a medium effect and CIs did not cross zero (Hedges' $g=0.60,95 \%$ CI $0.25,0.95$ ).

\section{Clinical outcomes related to pressure ulcers}

Seven $[22,24,25,29-31,33,34,36]$ of the ten RCTs measured skin status. Measurement methods varied (participant self-report [22, 29, 36], physical examinations carried out by HCPs [24, 25, 30, 33], HCP assessment of photos taken by patients/carer or patient self-report, and combined with verification of medical records [31, 34]). Participants' baseline skin status varied across studies (samples with and without PUs [25, 30, 31, 33], with both closed and open wounds [34], with a PU that had healed after surgical repair [22, 36], or PU-free [24]).

With the exception of one study [29], all studies reported some follow-up data on this outcome.

Only one intervention [22, 36] significantly improved skin status compared to controls. These results suggest that up to $4 \mathrm{~h}$ of structured education was more effective than the standard $1-2 \mathrm{~h}$ education program in preventing PU recurrence after surgical repair $[22,36](\mathrm{OR}=0.17,95 \% \mathrm{CI}$ $0.04,0.68)$. Three studies $[25,31,33,34]$ evaluating telehealth, self-management sessions plus motivational interviewing, and a risk assessment and feedback on PU risk factors' intervention did not find skin status variables (skin worsening, time to skin worsening, and PU development) to be significantly influenced (where calculated, OR 95\% CIs crossed 1). A significant subgroup analysis difference in PU development was noticed in the telehealth trial $[25,33]$ with intervention group females developing considerably fewer PUs than males at 6 months. The two last trials assessing PU prevalence and/or severity did not conduct statistical tests $[24,30]$. One [24] of these was a telehealth trial for which the OR 95\% CIs crossed 1, the other did not report the data clearly [30].

Only one study [34] measured health-care utilization for skin problems and number of days on bedrest. Results were reported incompletely, but health-care utilization was not significantly influenced by the intervention.

\section{Discussion}

To the best of our knowledge, this is the first study to examine the content of SCI skin care interventions using the BCT taxonomy. Twenty-eight of ninety-three defined BCTs were coded in 17 interventions. This is a slightly higher number of BCTs compared to those identified in a review of home-based rehabilitation interventions [40] that includes roughly the same number of studies, and a slightly smaller number than a review of interventions for chronic back pain and arthritis that includes 25 papers [41]. The number of
BCTs included in interventions is likely to vary according to patients' health behaviors, outcomes targeted, and the complexity of the medical condition. SCI is recognized to be very complex with several secondary complications to self-manage, which could explain the higher number of BCTs identified compared to other studies. The number of BCTs included may also depend on researchers' and clinicians' perceptions of what is required for behavior change. Some may still believe that behavior change can be achieved with simple knowledge-based interventions. Evidence suggests that increasing knowledge is necessary, but not sufficient, for behavior change [42]. PU prevention requires interventions that encourage routine performance of multiple skin care behaviors, develop patients' multidisciplinary knowledge, and include personalized intervention components. Repeated exposure to multiple BCTs delivered in different formats is likely required for behavior change and maintenance.

From a methodological perspective, the number of BCTs identified is related to the quality of intervention descriptions. The current study underlines the value of searching or contacting authors for intervention materials, as the number of BCTs coded using intervention materials was four times that of interventions for which none were available. The poor description of interventions in published materials underlines the need to report intervention content using published guidelines (e.g., Template for Intervention Description and Replication checklist [43]).

Sixty-five BCTs from the BCT taxonomy were not coded to any intervention, suggesting that many techniques from behavioral science remain unexplored despite their potential impact on skin care in SCI. Similar to other reviews on selfmanagement programs [40,41], the most commonly identified BCTs included instructions on how to perform behavior, credible source, and social support (unspecified). Testing other BCTs can advance the science of selfmanagement in SCI and our understanding of the underlying mechanisms through which they exert their effect. Not all BCTs in the BCT taxonomy may be applicable, feasible, or appealing in the SCI context (e.g., future punishment, pharmacological support, and paradoxical instructions). SCI researchers may benefit from conducting research to identify factors that influence skin care in SCI. These findings considered alongside a matrix of behavior change techniques linked to theoretical predictors of behavior [44] can help SCI researchers and clinicians select BCTs to include in skin care interventions. Factorial designs can be used to test multiple BCTs and can provide information on their individual and interaction effects.

The second aim of this study was to focus on RCTs to examine the effects of skin care self-management interventions on skin care and PU-related outcomes. Heterogeneous patient populations make RCTs difficult in 
rehabilitation research and the identification of 10 RCTs in this review is encouraging. Poor reporting, small sample sizes, variation in outcome measures and their psychometric properties, and mixed findings make it challenging to reach conclusions about the effects of the interventions reviewed. Effect sizes for the few studies and outcomes for which they could be calculated showed promise, particularly as their calculation was based on the longest follow-up when attrition rates tend to be highest. Reliance on shorter follow-up data would likely not influence findings as they were only available in one study [34]. Another finding from our review on intervention effectiveness suggests that wheelchair skills training programs that rely on patients selecting skills to work on are not ideal platforms through which to influence skin care, as patients prioritize everyday mobility skills. Making them mandatory to work on or a core component of these interventions is advised.

Skin status was the most commonly measured outcome yet comparisons across studies are difficult because of variation in measurement methods, length of follow-up, and baseline characteristics. Despite randomization, differences in skin status at baseline in particular can make results difficult to interpret if insufficient data are reported at follow-up on whether the PUs observed are new or the same as at baseline, their location, and their staging. Accurate and reliable PU measurements are recognized to be difficult [45]. It is recommended that researchers be precise in reporting data for this outcome, including detailed documentation of observed PU stages and anatomical locations [45]. This will enable reliable local and international benchmarking between health-care settings and facilities. The strong need for consistency in design and reporting was underlined by an International Guideline Recommendation Group [45], and it should be noted that there are internationally accepted standards available for reporting information about PUs in SCI [46]. In terms of study design, the majority of RCTs in this review included short-term follow-ups (6 months or less). Longer follow-ups are more likely to allow for behavioral and clinical changes. In addition, very few of the identified RCTs included more than one follow-up. Repeated assessments will help identify short- and long-term changes, and will allow for statistical analyses (e.g., mediation analyses) that investigate pathways of change.

Results of a posteriori analyses in one [25, 33] of the RCTs reviewed suggested that females benefitted from the intervention more than males. This may be related to male gender being a risk factor in PU development [47], or possibly to females responding better to self-management interventions [48]. Future work may benefit from planning such analyses during study design to ensure that gender interaction effects can be studied with sufficient statistical power.
Our risk of bias-assessment results suggests that much of the information required in papers to reach a judgment was unavailable ("uncertain" risk of bias assessment, Fig. 3). Researchers should use the Cochrane risk of bias tool to guide reporting on internal validity. Blinding of participants and personnel, and incomplete outcome data reporting were the two risk of bias domains most frequently rated "high risk". A discussion of the challenges to blind participants and personnel in behavioral interventions proposes some procedures to help reduce risk of bias [49]. Reporting of outcome data should not be influenced by the direction of the findings and incomplete reporting of outcome data should be justified.

Only three of the ten RCTs reviewed evaluated interventions delivered to community-dwelling people with SCI. High rates of PUs after discharge suggest that communitybased approaches are needed in PU prevention efforts. Networks of community care organizations and national registries can be used to reach people with a SCI in the community, and web-based technologies can be used to facilitate intervention delivery and receipt. In a Canadian survey [50], people with SCI confirmed the importance of developing community-based self-management programs, indicating that the Internet was the most appropriate delivery mode. In a separate survey on their information needs [51], medical issues relating to SCI (e.g., skin, bladder, bowel, and pain) ranked first. People with a new SCI diagnosis may be more receptive to preventive interventions after adjusting to life with SCI.

A limitation of this work is that non-randomized studies with one research group were not included in our review of intervention content. More inclusive inclusion criteria may have led to the identification of additional BCTs. In light of this, the consistency of our results with the number of BCTs identified in the number of BCTs identified in other reviews of self-management interventions is reassuring. Another limitation is that study characteristics made it difficult to conduct a meta-analysis or meta-regression to identify effective BCTs. In addition, descriptions of intervention components often did not specify the health behaviors they targeted (including skin care). Other reviews on physical activity and healthy eating [52] have included such analyses, likely because of the greater number of eligible studies, better reporting of intervention protocols and effects, and consistency in outcomes.

Strengths of this work include the robust search strategy and attempts to contact authors for intervention materials and outcome data. Our use of a standardized taxonomy of BCTs also contributes to building a science of selfmanagement in SCI by encouraging cross-study comparisons and common terminology in future studies.

This systematic review has identified the most commonly used BCTs in SCI skin care self-management interventions, 
as well as the potential promise of some of the tested SCI skin care interventions. Future work in this area would benefit from larger-scale studies, consistent use of validated outcome measures, and testing of a greater variety of BCTs.

Acknowledgements This work was funded through a postdoctoral fellowship granted by the Rick Hansen Institute. J.M.G. holds Canada Research Chair in Health Knowledge Uptake and Transfer.

Author contributions J.S.B., K.J.S., J.M.S., A.A., S.J., J.P., D.W., B. W., and J.G. were responsible for designing the systematic review protocol; J.S.B. was responsible for conducting the search; J.S.B. and J.M.S. were responsible for screening potentially eligible studies and J. G. for arbitrating potentially eligible studies; J.S.B. and K.J.S. were responsible for extracting and analyzing the data; J.S.B., K.J.S., J.M. S., A.A., S.J., J.P., D.W., B.W., and J.G. were responsible for interpreting results; J.S.B., K.J.S., and J.G. were responsible for designing and creating the results tables; J.S.B. drafted the manuscript; K.J.S., J. M.S., A.A., S.J., J.P., D.W., B.W., and JG provided feedback on the drafted manuscripts; J.S.B. and K.J.S. revised the manuscript; J.S.B., K.J.S., J.M.S., A.A., S.J., J.P., D.W., B.W., and J.G. approved the final version and agree to be accountable for all aspects of the work.

\section{Compliance with ethical standards}

Conflict of interest The authors declare that they have no conflict of interest.

Open Access This article is licensed under a Creative Commons Attribution 4.0 International License, which permits use, sharing, adaptation, distribution and reproduction in any medium or format, as long as you give appropriate credit to the original author(s) and the source, provide a link to the Creative Commons license, and indicate if changes were made. The images or other third party material in this article are included in the article's Creative Commons license, unless indicated otherwise in a credit line to the material. If material is not included in the article's Creative Commons license and your intended use is not permitted by statutory regulation or exceeds the permitted use, you will need to obtain permission directly from the copyright holder. To view a copy of this license, visit http://creativecommons. org/licenses/by/4.0/.

\section{References}

1. Brinkhof MW, Al-Khodairy A, Eriks-Hoogland I, Fekete C, Hinrichs T, Hund-Georgiadis M, et al. Health conditions in people with spinal cord injury: contemporary evidence from a populationbased community survey in Switzerland. J Rehabil Med. 2016;48:197-209.

2. White BAB, Dea N, Street JT, Cheng CL, Rivers CS, Attabib N, et al. The economic burden of urinary tract infection and pressure ulceration in acute traumatic spinal cord injury admissions: evidence for comparative economics and decision analytics from a matched case-control study. J Neurotrauma. 2017;34: 2892-900.

3. Gorecki C, Brown JM, Nelson EA. Impact of pressure ulcers on quality of life in older patients: a systematic review. J Am Geriatr Soc. 2009;57:1175-83.

4. van Loo MA, Post MW, Bloemen JH, van Asbeck FW. Care needs of persons with long-term spinal cord injury living at home in the Netherlands. Spinal Cord. 2010;48:423-8.
5. Bloemen-Vrencken J, Witte LP, Post MW, Heuvel WJ. Health behaviour of persons with spinal cord injury. Spinal Cord. 2007;45:243-9.

6. Knight K, Buchholz AC, Martin Ginis KA, Goy RE. Leisure-time physical activity and diet quality are not associated in people with chronic spinal cord injury. Spinal Cord. 2011;49:381-5.

7. Garber SL, Rintala DH, Rossi CD, Hart KA, Fuhrer MJ. Reported pressure ulcer prevention and management techniques by persons with spinal cord injury. Arch Phys Med Rehabil. 1996;77:744-9. Erratum. J Rehabil Res Dev. 2002;39(6)

8. Tung JY, Stead B, Mann W, Ba'Pham, Popovic MR. Assistive technologies for self-managed pressure ulcer prevention in spinal cord injury: a scoping review. J Rehabil Res Dev. 2015;52:131-46.

9. Cogan AM, Blanchard J, Garber SL, Vigen CLP, Carlson M, Clark FA. Systematic review of behavioral and educational interventions to prevent pressure ulcers in adults with spinal cord injury. Clin Rehabil. 2016;31:871-80.

10. Orenczuk KM, Mehta S, McIntyre A, Regan M, Teasell RW, SCIRE research team. A systematic review of the efficacy of pressure ulcer education interventions available for individuals with SCI. Can J Nurs Inform. 2011;6:3.

11. Michie S, Richardson M, Johnston $M$. The behavior change technique taxonomy (V1) of 93 hierarchically clustered with the number of BCTs identified in techniques: building an international consensus for the reporting of behavior change interventions. Ann Behav Med. 2013;46:81-95.

12. Baron J, Sullivan K, Swaine J, Aspinall A, Jaglal S, Presseau J, et al. Self-management interventions for skin care in people with a spinal cord injury: part 2-a systematic review of use of theory and quality of intervention reporting. Spinal cord. https://doi.org/ 10.1038/s41393-018-0136-5.

13. Baron J, Sullivan K, Swaine J, Presseau J, Aspinall A, Jaglal S, et al. Self-management interventions to improve skin care for pressure ulcer prevention in people with spinal cord injuries: a systematic review protocol. Syst Rev. 2016;5:150.

14. McGowan J, Sampson M, Salzwedel DM, Cogo E, Foerster V, Lefebvre C. PRESS peer review of electronic search strategies: 2015 guideline statement. J Clin Epidemiol. 2016;75:40-6.

15. Taylor SJC, Pinnock H, Epiphaniou E, Pearce G, Parke HL, Schwappach A, et al. A rapid synthesis of the evidence on interventions supporting self-management for people with longterm conditions: PRISMS - Practical systematic Review of SelfManagement Support for long-term conditions. Health Serv Deliv Res 2014;2.

16. Hedges L, Olkin I. Statistical methods for meta-analysis. Orlando, FL: Academic Press; 1985.

17. Higgins JPT, Altman DG, Gøtzsche PC, Jüni P, Moher D, Oxman $\mathrm{AD}$, et al. The Cochrane Collaboration's tool for assessing risk of bias in randomised trials. Br Med J. 2011;343:d5928.

18. Hossain MS, Harvey LA, Rahman MA, Muldoon S, Bowden JL, Islam MS, et al. Community-based InterVentions to prevent serIous Complications (CIVIC) following spinal cord injury in Bangladesh: protocol of a randomised controlled trial. BMJ Open. 2016;6:e010350.

19. Houlihan BV, Jette A, Paasche-Orlow M, Wierbicky J, Ducharme $\mathrm{S}$, Zazula $\mathrm{J}$, et al. A telerehabilitation intervention for persons with spinal cord dysfunction. Am J Phys Med Rehabil. 2011;90:756-64. 11

20. Norris W, Strickland, SB, Noble, CE SILS: spinal injury learning series. Mississippi Methodist Rehabilitation Center, Jackson: University Press of Mississippi; 1981.

21. Wyatt DWG. Reducing secondary conditions for spinal-cordinjured patients: pilot testing a risk assessment and feedback instrument. Top Spinal Cord Inj Rehabil. 2000;6:9-22. 
22. Garber SL, Rintala DH, Holmes SA, Rodriguez GP, Friedman J. A structured educational model to improve pressure ulcer prevention knowledge in veterans with spinal cord dysfunction. J Rehabil Res Dev. 2002;39:575-88.

23. Best KL, Miller WC, Huston G, Routhier F, Eng JJ. Pilot study of a peer-led wheelchair training program to improve self-efficacy using a manual wheelchair: a randomized controlled trial. Arch Phys Med Rehabil. 2016;97:37-44.

24. Hossain MS, Harvey LA, Rahman MA, Bowden JL, Islam MS, Taylor V, et al. A pilot randomised trial of community-based care following discharge from hospital with a recent spinal cord injury in Bangladesh. Clin Rehabil. 2016;31:781-9.

25. Houlihan BV, Jette A, Friedman RH, Paasche-Orlow M, Ni P, Wierbicky J, et al. A pilot study of a telehealth intervention for persons with spinal cord dysfunction. Spinal Cord. 2013;51:715-20.

26. Kennedy P, Berry C, Coggrave M, Rose L, Hamilton L. The effect of a specialist seating assessment clinic on the skin management of individuals with spinal cord injury. J Tissue Viability. 2003;13:122-5.

27. Ozturk A, Ucsular FD. Effectiveness of a wheelchair skills training programme for community-living users of manual wheelchairs in Turkey: a randomized controlled trial. Clin Rehabil. 2011;25:416-24.

28. Phillips VL, Temkin A, Vesmarovich S, Burns R, Idleman L. Using telehealth interventions to prevent pressure ulcers in newly injured spinal cord injury patients post-discharge. Results from a pilot study. Int J Technol Assess Health Care. 1999;15:749-55.

29. Phillips VL, Vesmarovich S, Hauber R, Wiggers E, Egner A. Telehealth: reaching out to newly injured spinal cord patients. Public Health Rep. 2001;116 (Suppl 1):94-102.

30. Rottkamp BC. An experimental nursing study: a behavior modification approach to nursing therapeutics in body positioning of spinal cord-injured patients. Nurs Res. 1976;25:181-6.

31. Rowland JL, White GW, Wyatt DA. Analysis of an intervention to reduce or prevent secondary conditions for people with spinal cord injuries. J Clin Psychol Med Settings. 2006;13:261-9.

32. Schopp LH, Clark MJ, Hagglund KJ, Mokelke EK, Stout BJ, Mazurek MO. Evaluation of a consumer-personal assistant training project. Disabil Rehabil. 2007;29:403-10.

33. Mercier HW, Ni P, Houlihan BV, Jette AM. Differential impact and use of a telehealth intervention by persons with MS or SCI. Am J Phys Med Rehabil. 2015;94:987-99.

34. Guihan M, Bombardier CH, Ehde DM, Rapacki LM, Rogers TJ, Bates-Jensen B, et al. Comparing multicomponent interventions to improve skin care behaviors and prevent recurrence in veterans hospitalized for severe pressure ulcers. Arch Phys Med Rehabil. 2014;95:1246-53.e3.

35. Norris WC, Vise GT Jr., Wharton GW, Noble CE, Atrickland SB. The spinal injury learning series: an experimental test. Arch Phys Med Rehabil. 1982;63:243-8.

36. Rintala DH, Garber SL, Friedman JD, Holmes SA. Preventing recurrent pressure ulcers in veterans with spinal cord injury: impact of a structured education and follow-up intervention. Arch Phys Med Rehabil. 2008;89:1429-41.

37. Scotzin M. A program design to motivate individuals with SCI for self-care. 98th Annual Meeting of the American Psychological Association; Boston, MA (1990).
38. Worobey LA, Kirby RL, Heinemann AW, Krobot EA, DysonHudson TA, Cowan RE, et al. Effectiveness of group wheelchair skills training for people with spinal cord injury: a randomized controlled trial. Arch Phys Med Rehabil. 2016;97:1777-84.e3.

39. Oberje EJ, Dima AL, Pijnappel FJ, Prins JM, de Bruin M. Assessing treatment-as-usual provided to control groups in adherence trials: exploring the use of an open-ended questionnaire for identifying behaviour change techniques. Psychol Health. 2015;30:897-910.

40. Heron N, Kee F, Donnelly M, Cardwell C, Tully MA, Cupples ME. Behaviour change techniques in home-based cardiac rehabilitation: a systematic review. Br J Gen Pract. 2016;66:e747-57.

41. Keogh A, Tully M, Matthews J, Hurley DA A review of behaviour change theories and techniques used in group based selfmanagement programmes for chronic low back pain and arthritis. Man Ther. 2015;20:727-735.

42. Ferris FD, von Gunten CF, Emanuel LL. Knowledge: insufficient for change. Int J Palliat Nurs. 2001;4:145-7.

43. Hoffmann TC, Glasziou PP, Boutron I, Milne R, Perera R, Moher $\mathrm{D}$, et al. Better reporting of interventions: template for intervention description and replication (TIDieR) checklist and guide. Br Med J. 2014;348:g1687.

44. French SD, Green SE, O'Connor DA, McKenzie JE, Francis JJ, Michie S, et al. Developing theory-informed behaviour change interventions to implement evidence into practice: a systematic approach using the Theoretical Domains Framework. Implement Sci. 2012;7:38

45. Haesler E, Kottner J, Cuddigan J. The 2014 International Pressure Ulcer Guideline: methods and development. J Adv Nurs. 2017;73:1515-30

46. Biering-Sorensen F, Alexander MS, van Asbeck FWA, Donovan W, Krassioukov A, Post MWM. Version 1.1 of the international spinal cord injury skin and thermoregulation function basic data set. Spinal Cord. 2017;55:566-9.

47. Eslami V, Saadat S, Habibi Arejan R, Vaccaro AR, Ghodsi SM, Rahimi-Movaghar V. Factors associated with the development of pressure ulcers after spinal cord injury. Spinal Cord. 2012;50:899-903.

48. Burner E, Menchine M, Taylor E, Arora S. Gender differences in diabetes self-management: a mixed-methods analysis of a mobile health intervention for inner-city Latino Patients. J Diabetes Sci Technol. 2013;7:111-8.

49. Friedberg JP, Lipsitz SR, Natarajan S. Challenges and recommendations for blinding in behavioral interventions illustrated using a case study of a behavioral intervention to lower blood pressure. Patient Educ Couns. 2010;78:5-11.

50. Munce SE, Fehlings M, Straus SE. Views of people with traumatic spinal cord injury about the components of selfmanagement programs and program delivery: a Canadian pilot study. BMC Neurol. 2014;14:209.

51. Matter B, Feinberg M, Schomer K, Harniss M, Brown P, Johnson $\mathrm{K}$. Information needs of people with spinal cord injuries. J Spinal Cord Med. 2009;32:545-54.

52. Samdal GB, Eide GE, Barth T, Williams G, Meland E. Effective behaviour change techniques for physical activity and healthy eating in overweight and obese adults; systematic review and meta-regression analyses. Int J Behav Nutr Phys Act. 2017;14:42. 